Enferm Bras 2019;18(4):544-51

https://doi.org/10.33233/eb.v18i4.2752

\title{
ARTIGO ORIGINAL \\ Caracterização do idoso vitimado pela violência no Pará
}

Thayse Moraes de Moraes, M.Sc.*, Salete Martens Aurelio**, Ana Maria Pantoja Costa Lamego* ${ }^{\star \star \star}$, Elizângela Fonseca de Mendonça ${ }^{* \star \star *}$, Lucia Hisako Takase Gonçalves, D.Sc. ${ }^{\star \star * \star *}$, Aline Bento Neves, M.Sc ${ }^{\star \star * \star \star *}$, Larissa de Cássia Pinheiro da Conceição***** Ana Rafaela Souza Rodrigues ${ }^{\star \star \star \star \star \star \star *}$

*Docente no curso de graduação em enfermagem pela Escola Superior da Amazônia (ESAMAZ) e Faculdade Integrada Brasil Amazônia (FIBRA), **Enfermeira, Especialista em Enfermagem em Terapia Intensiva, ${ }^{* \star \star}$ Enfermeira, Hospital Adventista de Belém, ${ }^{* \star * \star}$ Enfermeira, Especialista em Saúde Pública, Docente no curso de graduação em enfermagem pela Escola Superior da Amazônia (ESAMAZ), ${ }^{* * * * *}$ Enfermeira, Docente Titular aposentada da UFSC, Docente Visitante Sênior CAPES no PPGENF/UFPA, ${ }^{* \star * * \star \star}$ Enfermeira, ${ }^{* * * * * * \star}$ Acadêmica do curso de graduação em enfermagem, Escola Superior da Amazônia (ESAMAZ), ${ }^{* * * * * * \star}$ Enfermeira, Mestranda em Enfermagem pela Universidade Federal do Pará (UFPA)

Recebido 21 de janeiro de 2019; aceito em 1 de maio de 2019.

Correspondência: Thayse Moraes de Moraes, Passagem Mucajás, 63 A, Guamá CEP 66065332 Belém/PA

Thayse Moraes de Moraes: thaysemm@hotmail.com Salete Martens Aurelio: salete.martens@hotmail.com Ana Maria Pantoja Costa Lamego: analamego23@gmail.com Elizângela Fonseca de Mendonça: elizangelafm@yahoo.com.br Lucia Hisako Takase Gonçalves: Ihtakase@gmail.com

Aline Bento Neves: alinebentoneves@hotmail.com

Larissa de Cássia Pinheiro da Conceição: larissapc1939@gmail.com

Ana Rafaela Souza Rodrigues: anarafaela25portugal@gmail.com

\section{Resumo}

Objetivo: Caracterizar idosos submetidos à violência segundo as variáveis sociodemográficas no Estado do Pará no ano de 2014. Métodos: Trata-se de uma pesquisa de natureza descritiva, retrospectiva, documental, com abordagem quantitativa, realizada na Procuradoria de Justiça de Defesa da Pessoa com Deficiência e do Idoso e Acidente do Trabalho de Belém/PA. O processamento dos dados e a análise estatística foram realizados por meio do programa SPSS $\AA_{\text {, }}$ versão 22.0. Resultados: Os idosos, vítimas de violência cadastrados na Procuradoria de Justiça de Defesa da Pessoa com Deficiência e do Idoso e Acidente do Trabalho, no ano de 2014, eram majoritariamente do sexo feminino, viúvos e viúvas, pardos, tinham mais de 85 anos, eram provenientes da classe econômica $E$, residentes no município de Belém - a maioria reside com os filhos - e possuem casa própria. Quanto ao tipo de violência, a maioria sofreu negligência e os agentes agressores são os filhos, tendo sido os próprios membros da família os denunciantes da violência. Conclusão: Faz-se necessário que os serviços de defesa dos direitos e de proteção ao idoso sejam divulgados. É primordial a inserção do profissional enfermeiro no cotidiano da comunidade para auxiliar as famílias a enfrentarem os conflitos decorrentes de atos de violência. Palavras-chave: idoso, envelhecimento, violência, maus-tratos ao idoso, saúde do idoso, saúde pública, Enfermagem.

\section{Abstract \\ Characterization of elderly victims of violence in Amazonia}

Objective: To characterize older people submitted to violence according to sociodemographic variables in the state of Pará in 2014. Methods: A descriptive, retrospective and documentary study with a quantitative approach was conducted at the Prosecutor's Office for the Defense of Persons with Disabilities, the Aged and Occupational Accidents, in Belém/PA. Data were processed and statistical analysis was performed using SPSS®, version 18.0. Results: In 2014, older people who were victims of violence and registered at the Prosecutor's Office for the 
Defense of Persons with Disabilities, the Aged and Occupational Accidents were mainly female, belonging to the age group over 85 years old, widowed, of mixed ethnicity, economic class $\mathrm{E}$, residents of the municipality of Belém, mostly living with their adult children. Regarding the type of violence, most of them suffered negligence; had their own houses, and were assaulted by their children, with the family members themselves being the complainants. Conclusion: It is necessary to disseminate services for the defense of the rights and protection of older people. The insertion of nursing professionals in the community's day-to-day life is essential to help families face conflicts arising from acts of violence.

Key-words: aged, aging, violence, elder abuse, health of the elderly, public health, Nursing.

\section{Resumen}

\section{Caracterización del anciano víctima de violencia en Amazonía}

Objetivo: Caracterizar al anciano sometido a violencia según variables sociodemográficas en el Estado de Pará en 2014. Métodos: Investigación descriptiva, retrospectiva y documental con enfoque cuantitativo, realizada en la Fiscalía de Defensa de Personas con Discapacidad, del Anciano y de Accidentes de Trabajo, Belém, Pará. Datos procesados y análisis estadístico realizado mediante el programa SPSS $\AA$, versión 18.0. Resultados: Los ancianos víctimas de violencia registrados en la Fiscalía de Justicia para la Defensa de las Personas con Discapacidad, de los Ancianos y Accidentes de Trabajo, en 2014 eran, mayoritariamente, de sexo femenino, pertenecientes a la faja etaria mayor a 85 años, viudos (as), de etnia mestiza; clase económica $\mathrm{E}$, residentes en el municipio de Belém, mayoría residiendo con sus hijos. Respecto al tipo de violencia, la mayor parte sufrió negligencia; poseen domicilio propio, y el agente agresor son los hijos, siendo denunciantes los propios miembros de la familia. Conclusión: Resulta necesaria la difusión de los servicios de defensa de derechos y protección del anciano. La inserción del profesional de enfermería en el día a día comunitario es esencial para ayudar a las familias a enfrentarse a los conflictos derivados de actos de violencia.

Palabras-clave: anciano, envejecimiento, violencia, maltrato al anciano, salud del anciano, salud pública, Enfermería.

O rápido aumento da população idosa é um fenômeno mundial contemporâneo e essa transição demográfica tem ocorrido devido ao avanço no setor da saúde, à redução da taxa de natalidade resultante do declínio da taxa de fecundidade, da queda nas taxas de mortalidade e do aumento da expectativa de vida [1]. Em 2012, havia 810 milhões de pessoas idosas no mundo, o que equivale a $11,5 \%$ da população global, e há a estimativa de que, em 2050 , esse número chegue a dois bilhões, ou seja, $22 \%$ da população mundial será idosa. No Brasil, em 2005, havia cerca de 18 milhões de pessoas idosas, o que representava 9,9\% da população e estima-se que esse número chegue a 25 milhões em 2020 , atingindo $11,4 \%$ da população brasileira [2].

Segundo a Declaração de Toronto, em 2002, a violência contra o idoso foi descrita como um ato que pode configurar-se de forma isolada ou recorrente, ou ainda como ausência de uma solução propícia diante de um relacionamento em que haja confiança, tendo como implicação ao idoso, dano ou sofrimento [3]. O consenso internacional da Rede Internacional de Prevenção contra Maus-Tratos em Idosos define a violência contra esse grupo etário da seguinte forma: "O mau trato ao idoso é um ato (único ou repetido), ou omissão que lhe cause danos ou aflição e que se produz em qualquer relação na qual exista expectativa de confiança", no referido consenso foram estabelecidos pela Organização Mundial da Saúde (OMS) sete tipos de violências: abuso físico ou maus-tratos físicos, abuso ou maus-tratos psicológicos, a negligência, a autonegligência, o abandono, abuso financeiro, o abuso sexual, ato ou jogo sexual [4].

Estudo realizado a partir da base de dados Datasus, analisou os casos de idosos internados por agressões entre os anos de 2008 e 2013, considerando-se o Brasil como unidade geográfica de análise, e verificou que das 14.651 .626 internações de indivíduos com 60 anos ou mais no Brasil, 930.805 (6,3\%) ocorreram por causas externas, 16.814 (1,8\%) casos correspondiam a agressões atendidas em caráter de urgência ou em caráter eletivo. Tratandose de agressão corporal, de negligência e de abandono, essas violências foram a causa de, respectivamente, $1.787(10,6 \%)$ e $477(2,8 \%)$ internações de indivíduos idosos no período indicado [5].

No Brasil, necessita-se de mais estudos para se conhecer a magnitude do problema das agressões contra o idoso em virtude do caráter velado da violência. Os maus-tratos a idosos tornam-se uma preocupação generalizada de saúde pública com importantes consequências 
individuais, como a redução da sobrevida e os custos sociais [6]. Destaca-se que a literatura científica nacional e internacional tem descrito, como principais características das vítimas desse tipo de violência: sexo feminino [7-9], faixa etária entre 60- 80 anos [10], ser casado e morar acompanhado [11]. A adoção de comportamentos de promoção da saúde assim como intervenções educacionais podem ser eficazes na prevenção do abuso de idosos [12].

Nesse contexto, os profissionais de saúde têm papel relevante no enfrentamento da violência, na prevenção, na identificação precoce e/ou no cuidado ao idoso vitimado. Esta investigação objetivou caracterizar idosos submetidos à violência em relação às variáveis sociodemográficas no Estado do Pará no ano de 2014. Dessa forma, pretende-se contribuir tanto para a ampliação do conhecimento acerca da temática e quanto com programas e políticas públicas direcionadas aos idosos vítimas de violência.

Material e métodos

Trata-se de uma pesquisa de natureza descritiva, retrospectiva, documental, com abordagem quantitativa, realizada na Procuradoria de Justiça de Defesa da Pessoa com Deficiência e do Idoso e Acidente do Trabalho situada em Belém/PA.

A população fonte do estudo corresponde a 234 prontuários de ocorrências registradas por meio de denúncia de violência contra idosos no estado do Pará no ano de 2014. Posteriormente, foi realizado o cálculo amostral totalizando 146 ocorrências. Elegeu-se, como critérios de inclusão nesse recorte, ser idoso, isto é, ter 60 anos ou mais, ter sofrido alguma forma de violência e ter registrado as agressões no referido ano.

Realizou-se a coleta de dados entre janeiro e abril de 2016, na Procuradoria de Justiça de Defesa da Pessoa com Deficiência e do Idoso e Acidente do Trabalho. As fichas de atendimento aos idosos foram avaliadas e, a partir de então, realizou-se o preenchimento de um formulário, elaborado pelos autores, composto com as seguintes variáveis: aspectos sociodemográficos e econômicos - sexo, faixa etária, estado civil, etnia, município de ocorrência da agressão; classe socioeconômica, pertencimento da moradia, com quem a vítima morava no domicilio - e os dados relativos à denúncia de violência - o tipo de violência sofrida, o agente agressor, o denunciante.

O processamento dos dados e a análise estatística foram realizados por meio do programa SPSS $\AA$, versão 22.0. As variáveis quantitativas foram apresentadas por meio de média e desvio padrão e as qualitativas por meio de proporção, considerando-se um nível de significância de $5 \%$.

A pesquisa atendeu a todas as diretrizes da Resolução 466/2012 do Conselho Nacional de Saúde, foi encaminhada para apreciação e aprovação da Secretaria da Assistência Social e Cidadania e do Comitê de Ética em Pesquisa Curso de Graduação de Enfermagem da Faculdade Metropolitana da Amazônia (FAMAZ), tendo obtido o parecer de número 1.511.975.

Resultados

No estado do Pará, durante o período de 2014, notificaram-se 146 casos de idosos acometidos por violência, conforme mostra a Tabela I. Desse quantitativo, em 51 dos casos a vítima era do sexo masculino (34,93\%) e em $95(65,07 \%)$ dos casos, a vítima é do sexo feminino, havendo uma razão de 0,53 entre os sexos. No que se refere à faixa etária, observou-se que as vítimas foram os idosos acima de 85 anos, havendo 45 (30,83\%) casos registrados. Em relação à situação conjugal, $79(54,11 \%)$ dos idosos eram viúvos e viúvas, mas vale ressaltar que o quantitativo de vítimas casadas foi significativo, representando $20,55 \%$ do total, o que equivale a 30 casos.

Aproximadamente 70 idosos (47,95\%) eram de etnia parda. Quanto à procedência das denúncias, dos 144 municípios do Estado do Pará, apenas três municípios receberam notificação, sendo a grande maioria oriunda de Belém - 138 casos (94,53\%) -, Ananindeua - 6 casos $(4,10 \%)$ - e Vigia - 2 casos (1,37\%). Os demais municípios paraenses não receberam notificação de violência contra idoso no ano analisado.

Em relação à classe econômica, a maioria dos idosos pertence à classe $\mathrm{E}$ - 89 vítimas $(60,96 \%)$. Verificou-se que, em 88 casos $(60,28 \%)$, as casas em que as vítimas moravam pertenciam ao próprio idoso e, ainda, que $76(52,05 \%)$ dos idosos moravam com os filhos. 
Tabela I - Perfil socioeconômico e demográfico dos idosos da pesquisa/ Belém/PA, 2014.

\begin{tabular}{|c|c|c|c|c|}
\hline \multirow{2}{*}{\multicolumn{5}{|c|}{$\begin{array}{l}\text { Variáveis } \\
\text { Sexo }\end{array}$}} \\
\hline & & & & \\
\hline Masculino & 51 & & & 34,93 \\
\hline Feminino & 95 & & & 65,07 \\
\hline \multicolumn{5}{|c|}{ Faixa etária (anos) } \\
\hline $60-64$ & 11 & & & 7,53 \\
\hline $65-69$ & 12 & & & 8,22 \\
\hline $70-74$ & 18 & 79,26 & $60-90$ & 12,32 \\
\hline $75-79$ & 29 & & & 19,87 \\
\hline 80-84 & 31 & & & 21,23 \\
\hline$>85$ & 45 & & & 30,83 \\
\hline \multicolumn{5}{|l|}{ Estado civil } \\
\hline Solteiro & 22 & & & 15,07 \\
\hline Casado & 30 & & & 20,55 \\
\hline Divorciado & 12 & & & 8,22 \\
\hline Viúvo & 79 & & & 54,11 \\
\hline União estável & 3 & & & 2,05 \\
\hline \multicolumn{5}{|l|}{ Etnia } \\
\hline Branca & 62 & & & 42,47 \\
\hline Negra & 14 & & & 9,58 \\
\hline Parda & 70 & & & 47,95 \\
\hline \multicolumn{5}{|l|}{ Procedência } \\
\hline Belém & 138 & & & 94,53 \\
\hline Ananindeua & 6 & & & 4,1 \\
\hline Vigia & 2 & & & 1,37 \\
\hline \multicolumn{5}{|l|}{ Classe econômica } \\
\hline Classe A & 1 & & & 0,69 \\
\hline Classe B & 4 & & & 2,74 \\
\hline Classe C & 20 & & & 13,69 \\
\hline Classe D & 24 & & & 16,44 \\
\hline Classe E & 89 & & & 60,96 \\
\hline Não possui renda & 8 & & & 5,48 \\
\hline \multicolumn{5}{|c|}{ Pertencimento da moradia } \\
\hline Cônjuge & 2 & & & 1,37 \\
\hline Filhos & 28 & & & 19,18 \\
\hline Irmã (o) & 8 & & & 5,48 \\
\hline Própria & 88 & & & 60,28 \\
\hline Outros parentes & 8 & & & 5,48 \\
\hline Outros & 12 & & & 8,21 \\
\hline \multicolumn{5}{|c|}{ Com quem a vítima morava } \\
\hline Cônjuge & 13 & & & 8,9 \\
\hline Filhos & 76 & & & 52,05 \\
\hline $\operatorname{Irmã~(o)~}$ & 13 & & & 8,9 \\
\hline Neto & 7 & & & 4,8 \\
\hline Genro/Nora & 2 & & & 1,37 \\
\hline Própria & 21 & & & 14,38 \\
\hline Outros parentes & 7 & & & 4,8 \\
\hline Outros & 7 & & & 4,8 \\
\hline Total & 146 & & & 100 \\
\hline
\end{tabular}

Quanto ao tipo de violência, segundo a Tabela II, a maioria das vítimas sofreu negligência, o que aconteceu em 43 dos casos (29,45\%), seguidos de violência psicológica, em 31 casos $(21,24 \%)$. Ressalta-se que, em 94 das agressões $(64,39 \%)$, os agressores eram os próprios filhos dos idosos, sendo que, em 67 dos casos (4589\%), as denúncias foram efetuadas por algum filho que não é o agressor. 
Tabela II - Dados relativos à violência sofrida pelos idosos da pesquisa/ Belém/PA, 2014.

\begin{tabular}{lll}
\hline Variáveis & $\mathrm{n}^{\circ}$ & $\%$ \\
\hline Tipo de violência sofrida* & & \\
Física & 2 & 1,37 \\
Psicológica & 31 & 21,24 \\
Abandono & 13 & 8,9 \\
Negligência & 43 & 29,45 \\
Financeira, econômica e/ou patrimonial & 18 & 12,32 \\
Autonegligência & 17 & 11,64 \\
Física + psicológica + negligência & 1 & 0,69 \\
Física + psicológica & 13 & 8,9 \\
Negligência + financeira & 3 & 2,05 \\
Física + abandono & 1 & 0,69 \\
Abandono + negligência & 2 & 1,37 \\
Psicológica + financeira & 1 & 0,69 \\
Física + psicológica + financeira & 1 & 0,69 \\
\hline O agente agressor & & \\
Cônjuge & 1 & 0,69 \\
Filhos & 94 & 64,39 \\
Irmã (o) & 8 & 5,48 \\
Neto & 7 & 4,9 \\
Próprio idoso & 15 & 10,2 \\
Outros parentes & 17 & 11,64 \\
Outros & 4 & 2,7 \\
\hline O denunciante & & \\
Anônima & 22 & 15,06 \\
Cônjuge & 2 & 1,37 \\
Filhos & 67 & 45,89 \\
Irmã (o) & 7 & 4,79 \\
Neto & 1 & 0,69 \\
Genro/Nora & 1 & 0,69 \\
Vizinho & 5,43 \\
Própria & 17 & 11,64 \\
Outros parentes & 3,43 \\
Outros & 19 & 13,01 \\
\hline Porg do maipa & & \\
\hline & &
\end{tabular}

*Pergunta de múltipla escolha; Fonte: Autores.

Discussão

$\mathrm{Na}$ amostra estudada, houve predomínio de vítimas do sexo feminino, resultado similar a outros estudos encontrados na literatura nacional [7,13-14]. Quanto à faixa etária dos idosos agredidos, houve divergência relacionada a outro estudo, que foi realizado na cidade de Aracaju/SE, o qual mostra que as faixas etárias mais atingidas pela violência contra o idoso são: entre 60 e 69 (50,9\% dos casos analisados envolviam idosos nessa faixa etária), e entre 70 e 79 anos ( $27,7 \%$ dos casos analisados envolviam idosos nessa faixa etária). Essa diferença de dados encontrada entre alguns estudos remete à existência de expectativas de vida diferentes em cada região do país, assim, o presente estudo englobou uma faixa etária que corrobora estudos da região Norte. Estudos acrescentam que, quanto maior a idade, maior a vulnerabilidade do idoso, de modo que a idade é considerada outro fator de risco para violência em idosos $[1,15,16]$.

Estudo brasileiro aponta que avaliou a ocorrência de 224 boletins, verificou-se que 175 $(78,1 \%)$ denunciavam situações de maus-tratos, dentre os idosos vitimados $95(54,2 \%)$ eram viúvos, separados ou solteiros e $51(34,9 \%)$ eram casados/com companheiro [17], dados que condizem com os achados deste estudo. Apesar de haver predomínio da cor parda, na presente pesquisa, a etnia não foi considerada relevante como fator associado à violência, haja vista o Brasil ser um país de intensa miscigenação [1].

No que se diz respeito à procedência dos idosos, no presente estudo, a grande maioria das vítimas vem da capital paraense, totalizando 138 casos $(94,53 \%)$, e uma pequena quantidade é oriunda de Vigia $2(1,37 \%)$. Essa maior proporção de vítimas da capital em relação ao interior se deu em virtude da localização geográfica da Procuradoria de Justiça de Defesa da Pessoa com Deficiência e do Idoso e Acidente do Trabalho, o que torna o acesso, por parte dos 
idosos residentes na capital, mais fácil do que para os idosos do interior, que necessitam se deslocar para a instituição [7].

Percebe-se que $89(60,96 \%)$ dos idosos mais acometidos pela violência são aqueles que recebem até dois salários mínimos, isto é, que pertencem à classe $\mathrm{E}$, ou, ainda, aqueles que disponibilizam de baixos recursos financeiros, o que corrobora a ideia de que as características socioeconômicas estão vinculadas ao perfil das vítimas e de seus agressores [1].

Nas ilhas dos Açores em Portugal, foi realizado estudo referente à identificação dos fatores de risco em idosos que sofreram violência, $100 \%$ desses idosos não possuíam atividade laboral e 7,7\% não atingiram a renda mínima oficial [18]. Outro estudo mostrou dados sobre violência contra pessoas com 60 e mais anos residentes em Portugal há mais de 12 meses, caracterizando "Profissionais executantes de baixo rendimento", como aqueles que detinham o baixo rendimento $(67,2 \%)$, em que incluíam trabalhos que exigiam pouca qualificação, que trabalhavam ou tinham trabalhado por conta própria e escolaridade reduzida [11].

Em dois munícios do Nordeste brasileiro, mostrou a violência financeira como a segunda mais frequente, na qual a maioria da população idosa possuía baixa renda e recebiam benefícios sociais [19]. A violência financeira pode caracterizar-se por roubo ou utilização de objetos e/ou bens sem o consentimento do próprio idoso ou permissão, golpes e extorsão [20].

Em estudo realizado na atenção primária em Barcelona, na Espanha, investigou a detecção de maus-tratos em familiares que são cuidadores de idosos, o mesmo trouxe como achados que possíveis intervenções, como educação e treinamento, assistência financeira para casos de dependência, apoio social adequado e períodos de descanso para o cuidador, tais ações podem ter impactos significativos no bem-estar dos cuidadores e dos idosos que recebem esses cuidados, e poderiam propiciar um espaço para as famílias protegerem os idosos e diminuir o risco de maus-tratos [21].

Em relação ao número de moradores na mesma residência, confirmam-se os resultados de outros estudos. Verifica-se a relação do arranjo familiar com a violência doméstica, sobretudo quando há maior dependência, física ou mental, por parte dos idosos, ou quando esses apresentam maior grau de vulnerabilidade, e, nesse contexto, o convívio familiar estressante e cuidadores despreparados agravam a situação de violência [22]. Outro fator que pode intensificar a violência é quando o idoso tem sua autonomia prejudicada por doenças e os familiares adquirem a função de cuidadores [1,17].

No Brasil, houve uma redução da pobreza entre a população idosa: apenas $6 \%$ dela, atualmente, vive abaixo da linha de pobreza. Cerca de $25 \%$ dos aposentados conseguem se manter com três salários mínimos ou mais. A questão da pobreza na idade avançada é um dos fatores que influencia o aumento da dependência, produzida por condições físicas e psicológicas. Isso mostra que há uma relação entre dependência, pobreza e velhice, que pode influenciar na distribuição dos recursos econômicos individuais do idoso, e que é relevante para a elaboração e disponibilização de serviços sociais [23].

Observou-se, ainda, a predominância da negligência, violência denunciada em 43 dos casos analisados $(29,4 \%)$, seguida da violência psicológica, em 31 dos casos $(21,2 \%)$. Tais resultados são encontrados em outros estudos similares, nacional e internacionalmente $[10,20]$. A negligência consiste em ofertar ao idoso situações de risco para viver, a exemplo, morar em espaços sem condições de higiene adequada, sem segurança, sem cuidados referentes ao vestir, higiene e alimentação [11].

Os achados desta investigação mostram que a maioria dos agressores é membro da família, destacando-se os filhos dos idosos em 94 dos casos $(54,39 \%)$, o que caracteriza um predomínio da violência intrafamiliar. Frequentemente, o idoso tem dificuldade de denunciar 0 agressor por diferentes motivos: ele é, muitas vezes, um membro da família, a vítima constantemente insiste em defender e justificar as atitudes do seu agressor com medo de prejudicar seu filho, neto ou cuidador, ou com medo de as situações vividas piorarem. Há os casos em que os idosos não percebem o fenômeno como agressão ou violência. Dentro desse grupo de agressores, os filhos homens aparecem como sendo os mais frequentes $[3,13,14]$.

No que tange os denunciantes, neste estudo, predominaram os filhos que não eram os abusadores, resultado que difere de outros estudos recentes, que demonstram que as principais fontes de denúncias são anônimas - sendo que as fontes anônimas representam $50 \%$ dessas denúncias, as instituições de saúde, $21 \%$, e a família, 14\% [16].

O reconhecimento da notificação por parte do idoso como uma ferramenta de intervenção contra a violência é necessária [24]. Destaca-se a violência contra os idosos como um fenômeno sócio-histórico que afeta diretamente a saúde das vítimas, da família e da comunidade [25]. 
Ações conjuntas são necessárias para articulações entre entidades intersetoriais, tais como as unidades de saúde, a segurança pública, os centros comunitários e as entidades religiosas, entre outros [26]. Destaca-se que medidas de cuidados e de proteção praticadas desde os níveis primários de atenção à saúde poderiam contribuir significativamente para a redução dos coeficientes de mortalidade por agressões em idosos [25].

\section{Conclusão}

No município de Belém/PA, observa-se que a violência contra o idoso ocorre frequentemente sob as seguintes condições: as vítimas têm mais de 85 anos, são predominantemente do sexo feminino, viúvos e viúvas de etnia parda, originários da classe E. A maioria possuía casa própria e reside com os filhos, e esses são normalmente os agressores. Quanto ao tipo de violência, há prevalência da negligência e os denunciantes são os próprios membros da família. Nesse contexto, faz-se necessário que os serviços de defesa dos direitos e proteção ao idoso sejam divulgados, para que a comunidade tenha conhecimento de seus direitos e acesso a algum tipo de auxilio quando necessário.

Este estudo traçou o perfil de idosos que são vítimas de violência, podendo colaborar com estudos futuros nessa área além de contribuir com as políticas de saúde e de proteção da pessoa idosa. Algumas limitações se impuseram a esta pesquisa, sobretudo no que se refere ao uso de dados secundários - registros em prontuários - como base para inferências e análises, porém, isso não impossibilitou o alcance dos objetivos propostos.

É primordial a inserção do profissional enfermeiro nesse contexto e no cotidiano da comunidade para auxiliar as famílias a enfrentarem os conflitos decorrentes de atos de violência, para estimular e promover atividades educativas, de planejamento e de avaliação do cuidado ao idoso. $\mathrm{O}$ enfermeiro tem a responsabilidade de identificar a presença do evento da agressão e de denunciar a violência sofrida.

Referências

1. Aguiar MPC, Leite HA, Dias IM, Mattos MCT, Lima WR. Violência contra idosos: descrição de casos no Município de Aracaju, Sergipe, Brasil. Esc Anna Nery 2015;19(2):343-49. https://doi.org/10.5935/1414-8145.20150047

2. Vianna LG, Ferreira HHA, Duarte JA, De Oliveira BM, Cunha CS, Augusto NDA et al. A violência contra os idosos nos filmes. Revista Kairós Gerontologia 2015;18(2):167-95. https://revistas.pucsp.br/index.php/kairos/article/view/26499/18990

3. Organización Mundial de la Salud (OMS). Declaración de Toronto para la prevención Global del maltrato de las personas mayores. Ginebra: OMS; 2002.

4. World Health Organization (WHO). Missing voices: views folder persons on elder abuse. A study from eight countries: Argentina, Austria, Brazil, Canada, India, Kenya, Lebanon and Sweden. Geneva: WHO; 2002.

5. Castro VC, Rissardo LK, Carreira L. Violência contra os idosos brasileiros: uma análise das internações hospitalares. Rev Bras Enferm 2018;71(2):830-8. https://doi.org/10.1590/0034-7167-2017-0139

6. Pillemer K, Connolly MT, Breckman R, Spreng N, Lachs MS. Elder mistreatment: priorities for consideration by the white house conference on aging. Gerontologist 2015;55(2):320-7. https://doi.org/10.1093/geront/gnu180

7. Guimarães DBO, Mendes PN, Rodrigues IS, Feitosa CDA, Sales JCS, Figueiredo MLF. Caracterização da pessoa idosa vítima de violência. Rev Enferm UFPE on line 2016;10(Supl. 3):1343-50. https://doi.org/10.5205/reuol.7057-60979-3-SM1.1003sup201601

8. Guimarães APS, Górios C, Rodrigues CL, Armond JE. Notificação de violência intrafamilar contra a mulher idosa na cidade de São Paulo. Rev Bras Geriatr Gerontol 2018;21(1):91-7. https://doi.org/10.5205/reuol.7057-60979-3-SM-1.1003sup201601

9. Paraíba PMF, Silva MCM. Perfil da violência contra a pessoa idosa na cidade do Recife-PE. Rev Bras Geriatr Gerontol 2015;18(2):295-306. https://doi.org/10.1590/1809-9823.2015.14047

10. Paiva MM, Tavares DMS. Violência física e psicológica contra idosos: prevalência e fatores associados. Rev Bras Enferm 2015;68(6):1035-41. https://doi.org/10.1590/0034$\underline{7167.2015680606 \mathrm{i}}$ 
11. Gil AP, Santos AJ, Kislaya I, Santos C, Mascoli L, Ferreira Al, et al. Estudo sobre pessoas idosas vítimas de violência em Portugal: sociografia da ocorrência. Cad Saúde Pública 2015;31(6):1234-46. https://doi.org/10.1590/0102-311X00084614

12. Estebsari F, Dastoorpoor M, Mostafaei D, Khanjani N, Khalifehkandi ZR, Foroushani AR et al. Design and implementation of an empowerment model to prevent elder abuse: a randomized controlled trial. Clinical Interventions in Aging. Clin Interv Aging 2018;13:669-79. https://doi.org/10.2147/CIA.S158097

13. Mallet SDM, Cortes MDCJ W, Giacomin KC, Gontijo ED. Violência contra idosos: um grande desafio do envelhecimento. Rev Med Minas Gerais 2016;26(8):S408-S413. http://rmmg.org/artigo/detalhes/2188

14. Silva CFS, Dias AMSB. Violência contra idosos na família: motivações, sentimentos e necessidades do agressor. Psicologia: Ciência e Profissão 2016;36(3):637-52. https://doi.org/10.1590/1982-3703001462014

15. Brasil. Ministério do Planejamento. Orçamento e Gestão, Instituto Brasileiro de Geografia e Estatística. Mudança demográfica no Brasil no início do século XXI. Rio de Janeiro: IBGE; 2015. https://biblioteca.ibge.gov.br/visualizacao/livros/liv93322.pdf

16. Martins-Gil AP, Kislaya I, Santos AJ, Nunes B, Nicolau R, Fernandes AA. Elder abuse in Portugal: findings from the first national prevalence study. J Elder Abuse \& Neglect 2015;27(3):174-95. https://doi.org/10.1080/08946566.2014.953659

17. Irigaray TQ, Esteves CS, Pacheco JTB, Grassi-Oliveira R, Argimon IIL. Maus-tratos contra idosos em Porto Alegre, Rio Grande do Sul: um estudo documental. Estudos de Psicologia 2016;33(3):543-51. https://doi.org/10.1590/1982-02752016000300017

18. Carmona-Torres JM, Carvalhal-Silva RM, Vieira-Mendes MH, Recio-Andrade B, Goergen T, Rodríguez-Borrego MA. Elder abuse within the family environment in the Azores Islands. Rev Latinoam Enferm 2017;25:e2932. https://doi.org/10.1590/15188345.1871 .2932

19. Rodrigues RAP, Monteiro EA, Santos AMR, Ponte MLF, Fhon JRS, Bolina AF et al. Older adults abuse in three Brazilian cities. Rev Bras Enferm 2017;70(4):783-91. [Thematic Edition "Good Practices: Fundamentals of care in Gerontological Nursing"]. https://doi.org/10.1590/0034-7167-2017-0114

20. Mysyuk Y, Westendorp RG, Lindenberg J. How older persons explain why they became victims of abuse. Age Ageing 2016;45(5):696-702. https://doi.org/10.1093/ageing/afw100

21. Orfila F, Coma-Solé M, Cabanas M, Cegri-Lombardo F, Moleras-Serra A, Pujol-Ribera E. Family caregiver mistreatment of the elderly: prevalence of risk and associated factors. BMC Public Health 2018;18(1):167. https://doi.org/10.1186/s12889-018-5067-8

22. Oliveira KSM, Carvalho FPB, Oliveira LC, Simpson CA, Silva FTL, Martins AGC. Violência contra idosos: concepções dos profissionais de enfermagem acerca da detecção e prevenção. Rev Gaúcha Enferm 2018;39:e57462. https://doi.org/10.1590/1983-1447.2018.57462

23. Brasil. Secretaria de Direitos Humanos da Presidência da República. Secretaria de Direitos Humanos da Presidência da República. Manual de enfrentamento à violência contra a pessoa idosa. É possível prevenir. É necessário superar. Brasília, DF: Secretaria de Direitos Humanos da Presidência da República; 2013. http://www.sdh.gov.br/assuntos/pessoa-idosa/publicacoes/violencia-contra-a-pessoaidosa

24. Cachina AMP, Paiva IL, Torres TL. Violência intrafamiliar contra idosos: revisão sistemática. Liberabit 2016;22(2):185-96. http://www.scielo.org.pe/pdf/liber/v22n2/a06v22n2.pdf

25. Machado DR, Tavares RE, Tavares FG. Epidemiologia da mortalidade por agressões em idosos. Rev Enferm UFPE on line 2018;12(11):3015-23. https://doi.org/10.5205/1981-8963-v12i11a237176p3015-3023-2018

26. Rocha EM, Vilela ABA, Silva DM. Enfrentamento da violência intrafamiliar contra idosos pelos profissionais de saúde. Rev Kairós Gerontologia 2015;18(4):29-46. https://revistas.pucsp.br/index.php/kairos/article/viewFile/27567/19456 